\title{
An Ultra-Wideband Resistive-Feedback Low-Noise Amplifier with Noise Cancellation in $0.18 \mu \mathrm{m}$ Digital CMOS
}

\author{
Jianyun Hu, Yunliang Zhu, and Hui Wu \\ Laboratory for Advanced Integrated Circuits and Systems, \\ Department of Electrical and Computer Engineering, University of Rochester
}

\begin{abstract}
We present a wideband resistive feedback CMOS low-noise amplifier (LNA) with noise cancellation technique for ultra-wideband applications. The LNA achieves a 3-dB bandwidth of $0.7-6.5 \mathrm{GHz}$, power gain of $12.5 \mathrm{~dB}$, and noise figure of 3.5 - 4.2dB within the 3-dB bandwidth. The input matching is better than $-11 \mathrm{~dB}$ from 0.7 to $12 \mathrm{GHz}$. The IIP3 is measured $-5 \mathrm{dBm}$ at $5 \mathrm{GHz}$. It is implemented in a $0.18 \mu \mathrm{m}$ standard digital CMOS technology, occupies an area of $0.78 \mathrm{~mm} \times 0.68 \mathrm{~mm}$, and consumes $11.1 \mathrm{~mW}$ from a $1.8 \mathrm{~V}$ supply.
\end{abstract}

\section{INTRODUCTION}

Ultra-wideband (UWB) communications has become the one of the main research focus for both academia and industry with the approval of UWB technology for commercial applications in the $3.1-10.6 \mathrm{GHz}$ band by FCC [1]. With such a large bandwidth, UWB technologies promise to offer low-power, high data rate wireless connectivity for future short-range communication systems. UWB receivers (particularly UWB impulse radios) have relatively simple structures compare to their narrow-band counterpart. However, the large bandwidth presents new challenges for the front-end circuits such as low-noise amplifiers (LNA), correlators, and filters. Wideband front-ends are also needed for emerging multi-band, multistandard radio systems because of their lower cost, lower power, and better reconfigurability than conventional multi radio solutions [2].

There are many possible solutions for wideband LNAs [3], [4], [5], [6], as shown in Fig. 1. Distributed amplifiers [3] can provide very large bandwidth because of their unique gain-bandwidth trade-off. However, large power consumption and chip area make them unsuitable for typical low-power, low-cost UWB applications. Common-gate amplifiers [4], [7] exhibit excellent wideband input matching, but suffers from a relatively large noise figure (NF). Narrow-band LNAs like an inductively degenerated common-source amplifier can also be converted into a wideband one by adding a wideband input matching network [5]. However, the insertion loss of the passive input matching degrades the NF rapidly with frequency. Resistive-feedback amplifiers [6], [8], [9], [10] have very good wideband input matching characterisitc. However, low NF and low power consumption can be hardly achieved simultaneously across a large frequency range. In [11], noise cancellation technique is used to relax this trade-off in resistive-feedback amplifiers. In this paper, the bandwidth of a resistive-feedback amplifier with noise cancellation is further extended by using the inductive shunt peaking and series peaking techniques. Therefore, good input matching, low power consumption,

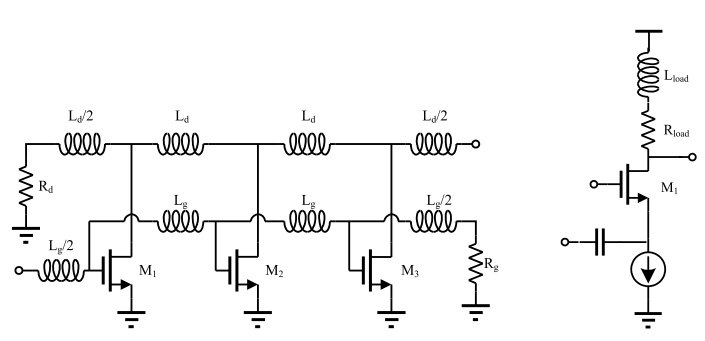

(a)

(b)

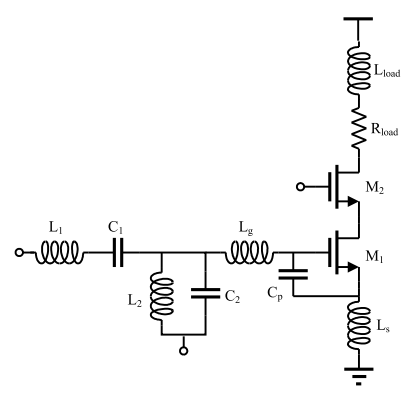

(c)

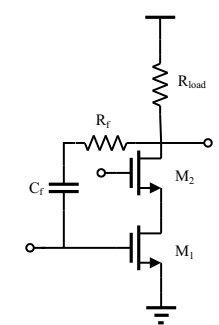

(d)
Fig. 1. Possible wideband LNA topologies: (a) Distributed amplifier; (b) Common-gate amplifier; (c) Passive input matching; and (d) Resistive feedback.

and low NF can be achieved simultaneously over a wide bandwidth.

\section{Circuit Analysis And Design}

The wideband resistive feedback noise canceling LNA is shown in Fig. 2. The first stage is based on resistive feedback LNA. The $L_{\text {load }}$ and $L_{g}$ are added for inductive shunt peaking and series peaking, respectively, which will be discussed later. The second stage $\left(M_{3}\right.$ and $M_{4}$, where $M_{3}$ acts as a source follower and $M_{4}$ acts as common-source amplifier) is added for wideband output matching and partially noise cancellation by connecting gate of $M_{4}$ with the gate of $M_{1}$.

Assume the main noise contribution of the LNA is from $M_{1}$, and its noise current is modeled by $i_{n}^{2}$. The noise current flows into the drain of $M_{2}$ (node X) through $M_{2}$ and part of it flows into input node (node Y) through $R_{f}$ and $C_{f}$. The noise voltage at node $\mathrm{X}$ and $\mathrm{Y}$ are fully correlated and have the same phases. These two noise voltages at node $\mathrm{X}$ and $\mathrm{Y}$ will appear 


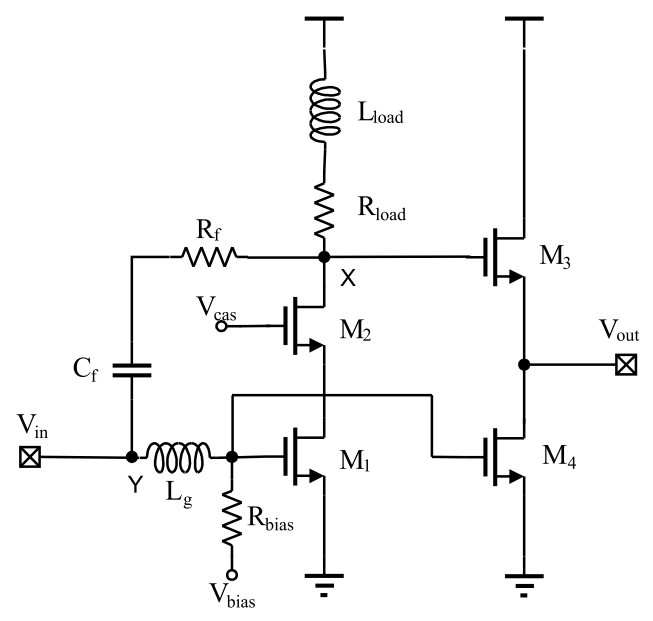

Fig. 2. Schematic of the wideband resistive-feedback noise-canceling LNA.

at output node through $M_{3}$ and $M_{4}$, respectively. The noise voltage at the source of $M_{3}$ keeps the same phase as node X, while the noise voltage at the drain of $M_{4}$ has the opposite phase from node Y. Therefore, the two noise voltages at output node are still correlated but with opposite phases. The noise current of $M_{1}$ can be partially or completed cancelled at the output node. It can be proved [11] that the noise current of $M_{1}$ can be completed cancelled if

$$
\frac{g_{m 4}}{g_{m 3}}=1+\frac{R_{f}}{R_{s}}
$$

where $g_{m 4}$ and $g_{m 3}$ are the transconductance of $M_{4}$ and $M_{3}$, respectively, and assumes source follower $M_{3}$ has a voltage gain of one.

While the noise can be partially or completely cancelled, the wanted signal can be added at the output node. The signal voltages at node $\mathrm{Y}$ and $\mathrm{X}$ have opposite phases. Therefore, after $M_{3}$ and $M_{4}$, the signal voltages at output node have same phases and will not be cancelled. Assuming source follower $M_{3}$ has unit voltage gain, then the overall voltage gain of the LNA is

$$
\begin{aligned}
A_{v}=\frac{V_{\text {out }}}{V_{\text {in }}} & =\frac{V_{X} A_{M 3}+V_{Y} A_{M 4}}{V_{Y}} \\
& =\frac{1-g_{M 1} R_{f}}{1+\frac{R_{f}}{R_{\text {load }}}}-\frac{g_{m 4}}{g_{m 3}}
\end{aligned}
$$

In the case of complete noise cancellation, the overall voltage gain of the LNA is

$$
A_{v, n c}=\frac{1-g_{M 1} R_{f}}{1+\frac{R_{f}}{R_{\text {load }}}}-1-\frac{R_{f}}{R_{s}}
$$

Assuming complete noise cancellation and neglecting noise contribution of the cascade device $M_{2}$ and series inductor $L_{g}$, the noise factor after first stage is mainly determined by $R_{s}$, $R_{f}$ and $R_{\text {load }}$. Here the noise contribution by $L_{\text {load }}$ can be

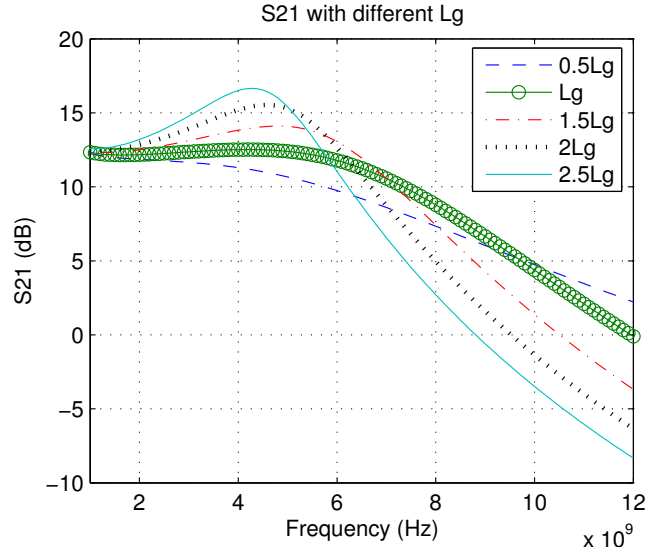

Fig. 3. Power gain versus frequency with different $L_{g}$.

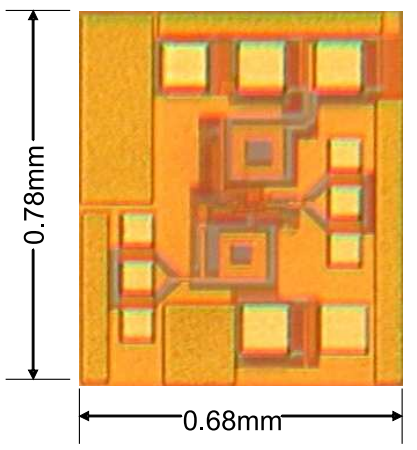

Fig. 4. Chip photo of the prototype LNA.

included in that by $R_{\text {load }}$. The noise factors of first stage can be derived [4]

$$
F_{1}=1+\frac{R_{f}\left(1+g_{M 1} R_{s}\right)^{2}}{R_{s}\left(1-g_{M 1} R_{f}\right)^{2}}+\frac{\left(R_{s}+R_{f}\right)^{2}}{R_{s} R_{\text {load }}\left(1-g_{M 1} R_{f}\right)}
$$

where the second and third term are the noise contribution by $R_{f}$ and $R_{\text {load }}$, respectively. The noise factors at the output node can be derived as

$$
F_{t}=1+\frac{R_{s}\left(\frac{R_{s}+R_{f}}{R_{\text {load }}}+1+g_{M 1} R_{s}\right)^{2}}{R_{f}\left(\frac{R_{s}+R_{f}}{R_{\text {load }}}-1-g_{M 1} R_{s}\right)^{2}}
$$

where the second term is the noise contribution by $R_{f}$. The noise contribution by $M_{3}$ and $M_{4}$ are ignored here, since they are very small compared to that by $R_{f}$. As is shown in (6), in addition to the noise contribution from $M_{1}$, the noise contribution from $R_{\text {load }}$ can also be cancelled at the output node, which can further lower the total noise factor.

The inductor $L_{\text {load }}$ of the LNA is used for shunt peaking purpose at high frequency. The inductive load can provide a resonant peaking at the output when the amplifier starts to roll off at high frequencies and equalize the power gain of the LNA to a constant value across the bandwidth by compensating the decreasing impedance of capacitance with the increase 


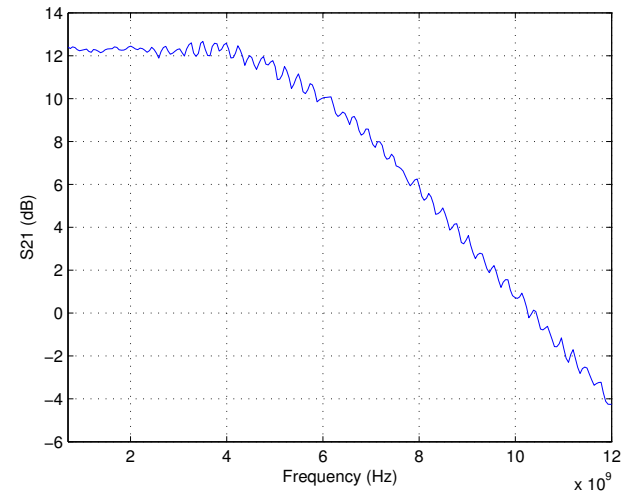

Fig. 5. Measured power gain.

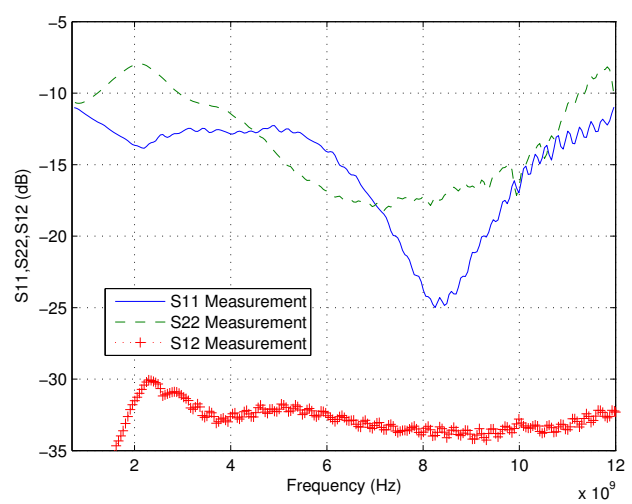

Fig. 6. Measured return loss and reverse isolation.

of frequency [12]. The series inductor $L_{g}$ is used to further boost the gain at high frequencies and extend the bandwidth by resonating with the parasitic gate to source capacitance of $M_{1}$ and parasitic capacitance formed by bottom plate of $C_{f}$ to substrate[13][14]. Fig. 3 shows the simulated power gain with different value of $L_{g}$. As is shown in the Fig. 3, a higher gain and larger bandwidth can be achieved with an optimized $L_{g}$. In addition to the gain boost and bandwidth extension, the resonance between $L_{g}$ and the parasitic gate to source capacitance of $M_{1}$ and parasitic capacitance formed by bottom plate of $C_{f}$ to substrate can benefit the input matching, high gain and low noise. As is shown in [4][5], resistive feedback LNA suffers from the tradeoff between input matching, gain and noise figure. The use of the $L_{g}$ to resonate with the parasitic capacitance at input node relaxes of the tradeoff and can achieve the good input matching, high gain and low noise simultaneously.

\section{MEASUREMENT RESULTS}

The wideband resistive feedback LNA is designed and implemented in a standard $0.18 \mu \mathrm{m}$ digital CMOS process. The microphotograph of this LNA is shown in Fig. 4. The chip occupies an area of $0.78 \mathrm{~mm} \times 0.68 \mathrm{~mm}$. The LNA consumes $11.1 \mathrm{~mW}$ at a voltage supply of $1.8 \mathrm{~V}$.

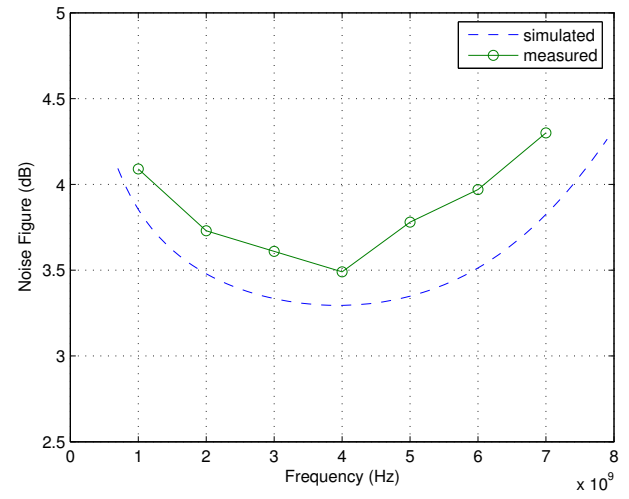

Fig. 7. Measured and simulated noise figure.

Fig. 5 shows the measured power gain of the LNA. The measured power gain achieves a maximum of $12.5 \mathrm{~dB}$ and the $3-\mathrm{dB}$ bandwidth is $0.7-6.5 \mathrm{GHz}$. The LNA remains 1$\mathrm{dB}$ flatness from 0.7 to $4.5 \mathrm{GHz}$.

The measured input, output return loss and the measured reverse isolation are presented in Fig. 6. The input return loss is better than $-11 \mathrm{~dB}$ in the $3-\mathrm{dB}$ bandwidth and remains the performance up to $12 \mathrm{GHz}$. The output return loss is below $-8 \mathrm{~dB}$ up to $12 \mathrm{GHz}$ and remains a value of less than $-10 \mathrm{~dB}$ across the most of the band. As is shown in Fig. 6, the measured reverse isolation is below $-30 \mathrm{~dB}$ across the band of interest.

The measured and simulated noise figures are illustrated in Fig. 7. The measured noise figure is from 3.5 to $4.2 \mathrm{~dB}$ across the $3-\mathrm{dB}$ bandwidth, which is slightly higher than the simulated noise figure. This is mainly due to the lack of very accurate noise model of deep submicron CMOS. The measured and simulated noise figures show the good effect of the wideband noise cancellation on the LNA.

The measured IIP3 with two-tone test is shown in Fig. 8 and Fig. 9. The measurement is performed at $1 \mathrm{GHz}, 3 \mathrm{GHz}$ and $5 \mathrm{GHz}$. As is shown in Fig. 8 and Fig. 9. The measured IIP3 is $-5.8 \mathrm{dBm},-5.9 \mathrm{dBm}$ and $-5 \mathrm{dBm}$ at $1 \mathrm{GHz}, 3 \mathrm{GHz}$ and $5 \mathrm{GHz}$, respectively.

Performance is summarized in Table I. Comparisons with the previously reported LNAs are also listed in Table I. Compared to the previously published LNAs, especially the wideband resistive feedback LNAs, this work achieves good input matching, low noise figure and low dc current consumption across a wide range of frequencies simultaneously.

A figure of merit (FOM) is used here to compare the performance of different LNAs with similar function. The FOM here evalutes the maximum power gain, 3-dB bandwidth, excess noise factor and the power consumption of the LNA and it is defined as

$$
F O M=\frac{|S 21| B W[G H z]}{|F-1| \operatorname{Power}[m W]}
$$

Based on the calculated FOM in Table I, this work has a 
Table I. Performance Summary

\begin{tabular}{|c|c|c|c|c|c|c|c|c|c|}
\hline Ref. & $\begin{array}{c}\text { CMOS } \\
\text { Tech. }\end{array}$ & $\begin{array}{l}\text { Bandwidth } \\
(\mathrm{GHz})\end{array}$ & $\begin{array}{l}\mathrm{S} 21_{\max } \\
(\mathrm{dB})\end{array}$ & $\begin{array}{l}\mathrm{S} 11_{\max } \\
(\mathrm{dB})\end{array}$ & $\begin{array}{l}\mathrm{NF} \\
(\mathrm{dB})\end{array}$ & $\begin{array}{c}\text { IIP3 } \\
(\mathrm{dBm})\end{array}$ & $\begin{array}{l}\text { Supply } \\
\text { (V) }\end{array}$ & $\begin{array}{l}\text { Power } \\
(\mathrm{mW})\end{array}$ & FOM \\
\hline [6] & $0.13-\mu \mathrm{m}$ & 5.9 & 16 & -9 & $4.7-5.7$ & - & 2 & $38^{*}$ & 3.2 \\
\hline [8] & $0.18-\mu \mathrm{m}$ & $2-4.6$ & 9.8 & -9 & $2.3-5.2$ & $-7 @ 4 \mathrm{GHz}$ & 1.8 & 12.6 & 2.8 \\
\hline [9] & $0.13-\mu \mathrm{m}$ & $1-7$ & 17 & -10 & 2.4@3GHz & -4.1 & 1.4 & $25^{*}$ & 16.3 \\
\hline [10] & 90-nm & $0.4-1$ & 16 & -10 & $3.5-5.3$ & -17@0.9GHz & 1.2 & 16.8 & 1.1 \\
\hline [11] & $0.25-\mu \mathrm{m}$ & $0.002-1.6$ & 13.7 & -8 & $1.9-2.4$ & $0 @ 0.9 \mathrm{GHz}$ & 2.5 & 35 & 1.9 \\
\hline [7] & $0.13-\mu \mathrm{m}$ & $0.8-2.1$ & 14.5 & -8.5 & 2.6 & $16 @ 0.9,2 \mathrm{GHz}$ & 1.5 & 11.6 & 3.9 \\
\hline This Work & $0.18-\mu \mathrm{m}$ & $0.7-6.5$ & 12.5 & -11 & $3.5-4.2$ & $-5 @ 5 \mathrm{GHz}$ & 1.8 & 11.1 & 7.5 \\
\hline
\end{tabular}

*Differential outputs.

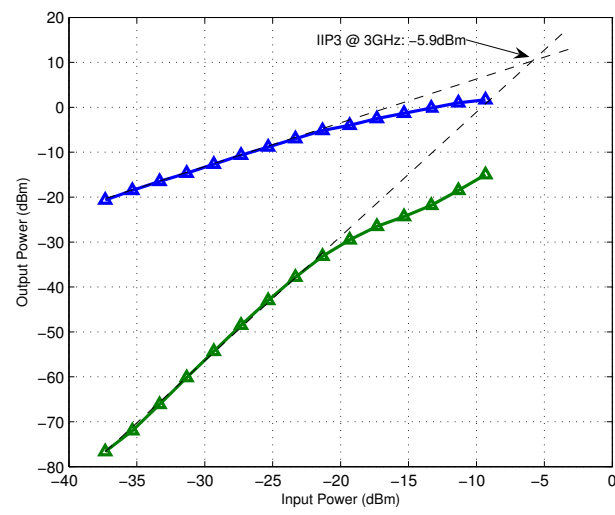

Fig. 8. Measured IIP3 at $3 \mathrm{GHz}$.

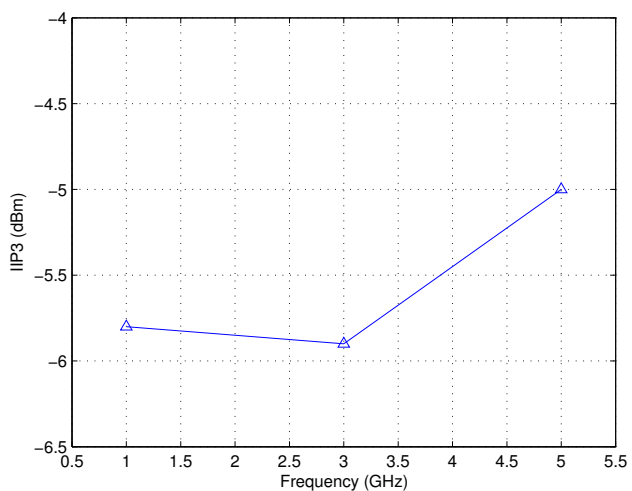

Fig. 9. Measured IIP3 at different frequencies.

good performance and is better than all the other works expect for [9].

\section{CONCLUSION}

A wideband resistive feedback CMOS LNA with noise cancellation for multi-band, multi-standard receivers is implemented in $0.18 \mu \mathrm{m}$ standard digital CMOS process. By using the design technique for wideband LNA, the proposed LNA achieves a power gain of $12.5 \mathrm{~dB}$, a noise figure from 3.5 to $4.2 \mathrm{~dB}$ across the $3-\mathrm{dB}$ bandwidth from 0.7 to $6.5 \mathrm{GHz}$. The input return loss is better than $-11 \mathrm{~dB}$ up to $12 \mathrm{GHz}$. The
LNA occupies an area of $0.78 \mathrm{~mm} \times 0.68 \mathrm{~mm}$, and consumes $11.1 \mathrm{~mW}$ from a voltage supply of $1.8 \mathrm{~V}$.

\section{ACKNOWLEDGMENT}

The authors would like to thank Bijoy Chatterjee, Ahmad Bahai, Peter Holloway, Mounir Bohsali, Johnny Yu, Anish Shah, Virginia Abellera, Peter Misich, and Jun Wan of National Semiconductor for their help and support in chip fabrication.

\section{REFERENCES}

[1] Revision of part 15 of the commission's rules regarding ultra-wide-band transmission systems. Federal Communication Commission, Washington, DC, First Report and Order, 2002.

[2] S. Chehrazi, A. Mirzaei, R. Bagheri, and A. A. Abidi. A $6.5 \mathrm{GHz}$ wideband CMOS low noise amplifier for multiband use. In Proc. IEEE Custom Integrated Circuits Conference, pages 801-804, 2005.

[3] B. M. Ballweber, R. Gupta, and D. J. Allstot. A fully integrated 0.55.5-GHz CMOS distributed amplifier. IEEE J. Solid-State Circuits, 35(2):231-239, Feb. 2000.

[4] C.-F. Liao and S.-I. Liu. A broadband noise-canceling CMOS LNA for 3.1-10.6-GHz UWB receivers. IEEE J. Solid-State Circuits, 42(2):329_ 339, Feb. 2007.

[5] A. Bevilacqua and A. M. Niknejad. An ultra wide-band CMOS LNA for 3.1-10.6 GHz wireless receivers. In ISSCC Dig. Tech. Papers, pages 382-533, 2004.

[6] R. Gharpurey. A broadband low-noise front-end amplifier for ultra wideband in 0.13um CMOS. IEEE J. Solid-State Circuits, 40(9):19831986, Sep. 2005.

[7] W. H. Chen, G. Liu, B. Zdravko, and A. M. Niknejad. A highly linear broadband CMOS LNA employing noise and distortion cancellation. In IEEE RFIC Symp. Dig. Papers, pages 61-64, 2007.

[8] C.-W. Kim, M.-S. Kang, P. T. Anh, H.-T. Kim, and S.-G. Lee. An ultrawideband CMOS low noise amplifier for $3-5 \mathrm{GHz}$ UWB system. IEEE J. Solid-State Circuits, 40(2):544-547, Feb. 2005.

[9] R. Ramzan, S. Andersson, J. Dabrowski, and C. Svensson. A 1.4V $25 \mathrm{~mW}$ inductorless wideband LNA in 0.13um CMOS. In ISSCC Dig. Tech. Papers, pages 424-425, 2007.

[10] M. Vidojkovic, M. Sanduleanu, J. V. D. Tang, P. Baltus, and A. V. Roermund. A $1.2 \mathrm{~V}$, inductorless, broadband LNA in 90nm CMOS LP. In IEEE RFIC Symp. Dig. Papers, pages 53-56, 2007.

[11] F. Bruccoleri, E. A. M. Klumperink, and B. Nauta. Noise canceling in wideband CMOS LNAs. In ISSCC Dig. Tech. Papers, pages 406-407, 2002.

[12] A. Ismail and A.A. Abidi. A 3-10-GHz low-noise amaplifier with wideband LC-ladder matching network. IEEE J. Solid-State Circuits, 39(12):2269-2277, Dec. 2004.

[13] Y. Gao, Y. Zheng, and B.-L. Ooi. A 0.18-um CMOS UWB LNA with $5 \mathrm{GHz}$ interference rejection. In IEEE RFIC Symp. Dig. Papers, pages 47-50, 2007.

[14] S. Galal and B. Razavi. 40Gb/s amplifier and ESD protection circuit in 0.18um CMOS technology. In ISSCC Dig. Tech. Papers, pages 480-481, 2004. 